alopecia. The severe pain radiates along the course of the supraorbital nerve when it is stimulated on pressure. The supraorbital causalgia is caused by direct traumas (bruise, laceration, fracture) to its nerve fibere and artery comming out to the subcutaneous tissue through the supraorbital foramen, and it may occure when the hyperflexion of the supraorbital nerve is resulted from the upward traction at the site of the foramen without direct trauma. Surgical treatments for the syndrom are procain block or neurectomy of the supraorbital nerve.

\title{
37. Psychological Responses and Autonomic Nerve Functions in Cases of Headache
}

\author{
Noboru Hozumi, Yasuo UNaI and Chikako Fukatsu \\ Department of Neuropsychiatry, Keio Gijuku University \\ School of Medicine
}

In our previous study we observed a positive correlation between urine copo excretion and the autonomic nerve function, and also saw that a large amount of copo is excreted in some cases of headache. By this, we are led to the hypothesis that some disorder of the autonomic nerve center may precede the physical state which is liable to produce headache.

Thirty-two patients were tested according to the Askevold method, slightly modified, to measure the amount of copo excretion and given the Mechollyl test to measure the function of the autonomic nerves. In addition to these, the Fukamachi's CMI, SCT and TPI tests were used to obtain data on psychological state of the patients.

The above hypothesis was tested in two ways: First, a significant difference between the amount of urine copo excreted by these patients and the amount in normal persons was observed at the $.1 \%$ level on the T-test. A comparison of the amount of urine copo excreted before and after the Mechollyl test between the two groups showed a similar general tendency to increase, but the migraine patients of the P-type showed a tendency of tension headache of the same type. The critical ratio on the assumption that all migraine patients of tension headache can be categorized as the Ptype, was calculated and found out as below the 1\% level.

Secondary, the results of the psychological tests were collelated and seemed to indicate that one half of patients of migraine and of tension headache were neurotic or psychologically unstable. From all the above evidence, 
therefore, it may be safe to conclude that the results strongly supported the the above hypothesis.

Eventually, the same results also confirmed a hypothesis of Schumacher, et al. 1941 that migraine is primarily related to an unstable state of the autonomic nerve, and related to also psychological factors caused by imbalance of blood distribution localized at the base of brain.

(copo: coproporphyllin)

\title{
38. Neurolept-Analgesia in Intracranial Surgery
}

\author{
Mitsuo Satoh, Mitsuko Satoyoshi, Keiji Kaya, Ikuo Yamanaka, \\ Ryohei KaWAmura and Tomomichi Hiraiwa \\ Dept. of Anesthesiology, Juntendo University, School of Medicine
}

So-called neurolept-analgesia for the intracranial operations was successfully obtained by the intravenous administration of the Mixture 1, contained pethilorfan, hydergine, vesprine and atropine and of the Mixture 2, using gamma-hydroxybutyrate or butyrolactone, with supplementary use of local or light general nitrous oxide-oxygen anesthesia.

As compared with fluothane anesthesia, the respiratory, cardiovascular and metabolic changes were markedly reduced by neurolept-analgesia, though with spontaneous respiration slight tendency to respiratory acidosis was occasionally observed in the blood gas analysis.

Post-operative recovery was more rapid and uneventful. Only in some instances, retching and restlessness were observed with the Mixture 2 immediately after the operation.

\section{Histological Study on Cranial Sutures of Secondary Craniostenosis}

\author{
Susumu MrYataka, Shozo Yodóshi, Shozaburo Utsumi, \\ and Yutaka HoRI \\ 2nd Dept. of Surgery, Nara Medical College
}

In our clinic, it has been found $20 \%$ of cerebral palsied patients with craniostenotic states in their skull and sutures which craniotomy are effective. 